\title{
Myocardial infarction caused by Graves' disease
}

\author{
Marek Kuch, Magdalena Rostek, Marek Chmielewski, Monika Bargieł \\ Department of Cardiology, Arterial Hypertension, Second Faculty of Medicine, Medical University of Warsaw, \\ Mazovian Bródno Hospital, Warsaw, Poland
}

Key words: coronary artery spasm; thyrotoxicosis; myocardial infarction; Graves' disease

Coronary artery spasm is a rare cause of myocardial infarction ( $0.5 \%$ of cases). It can also be complicated by dysrhythmia or sudden cardiac death. We present a case of a 41-year-old woman with non-ST elevation myocardial infarction caused by coronary artery spasm associated with hyperthyroidism. The coronarography showed a spasm of the left cardiac artery, which was completely relieved by administration of intracoronary nitrate. In the course of the further research the patient was diagnosed with Graves' disease.

A 41-year-old female patient with a history of arterial hypertension and smoking was admitted to the Cardiology Department with severe chest pain remittent for 3 days at rest. ECG showed a sinus tachycardia and features of cardiac muscle ischaemia - ST segment depression on anterior, inferior, and lateral leads and elevation in augmented vector right (aVR) (Fig. 1). The laboratory tests showed elevated markers of myocardial injury — high-sensitivity cardiac troponin I (hs-cTnI: 150 ng/L; ref. range < 16 ng/L). A coronary angiography was urgently performed. It visualised a spasm of the trunk of the left coronary artery constricting the vessel by $70 \%$. No significant stenosis was found in the other arteries (Fig. 2 and 3). After the procedure the patient was transferred to the Cardiac Intensive Care Unit. The chest pain completely subsided. A physical examination revealed higher body temperature, persistent tachycardia, symmetrical exophthalmia, and tremor. In

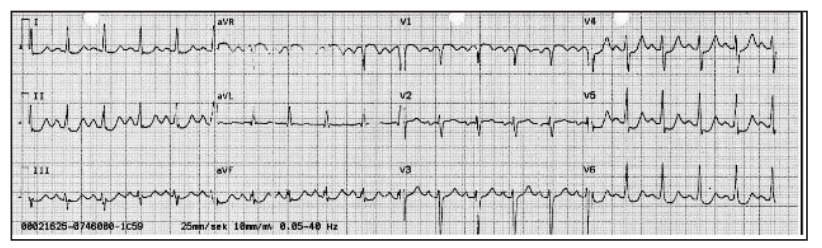

Figure 1. Electrocardiogram of the patient addition, the patient lost $20 \mathrm{~kg}$ of body weight within 6 months. Until hospitalisation the patient had no history of thyroid disease. The laboratory tests revealed features of hyperthyroidism - undetectable concentration of thyroid-stimulating hormone (TSH: $0.0 \mathrm{uIU} / \mathrm{mL}$; ref. range $0.35-4.0 \mathrm{uIU} / \mathrm{mL}$ ) and significantly elevated concentrations of free thyroid hormones (fT3: 20.84 $\mathrm{pg} / \mathrm{mL}$; ref. range 1.71-3.71 pg/mL, fT4: $2.76 \mathrm{ng} / \mathrm{dL}$; ref. range $0.71-48 \mathrm{ng} / \mathrm{dL}$ ). Immunoassays showed elevated concentrations of antibodies against TSH receptor (TRAb: $13.73 \mathrm{U} / \mathrm{L}$; ref. range $0-1.5 \mathrm{U} / \mathrm{L}$ ), antibodies against thyreoglobulin $(32.24 \mathrm{IU} / \mathrm{mL}$; ref. range $<4.1$ $\mathrm{IU} / \mathrm{mL}$ ), and an abnormally high concentration of anti-thyroid peroxidase antibodies (ATPO > 1000.0 $\mathrm{IU} / \mathrm{mL}$; ref. range $0-5.61 \mathrm{IU} / \mathrm{mL}$ ). USG showed parenchymatous goitre without nodule with decreased echogenicity. Echocardiography performed after the coronarography showed enlarged left ventricle of 6.3 $\mathrm{cm}$ (ref. range $<5.5 \mathrm{~cm}$ ) and hyperdynamic circulation features. Left ventricular ejection fraction was $70 \%$. No segmental contractility disorders were observed. Based on the tests results, Graves' disease was diagnosed. The patient was successfully treated with diltiazem, nitroglycerin, and thiamazole.

Coronary artery spasm, especially when caused by thyrotoxicosis, is a rare cause of acute coronary syndrome. This case highlights the importance of considering hyperthyroidism in the differential diagnosis of chest pain with normal coronary arteries. Thyroid function testing should be routinely performed on a patient with coronary artery spasm, especially for young female patients without typical risk factors of coronary heart disease. According to the reports, vasospastic angina may be involved in up to $20 \%$ of patients with thyrotoxicosis. The exacts mechanisms of artery spasm connected with thyrotoxicosis are unclear. In vitro studies have proven a trend of enhanced vasoconstric- 


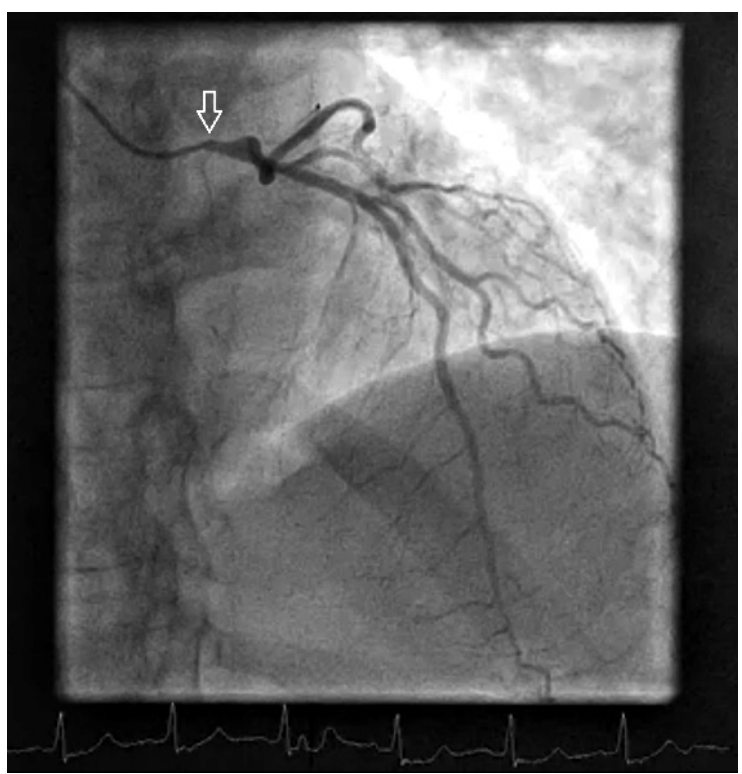

Figure 2. Coronary angiogram of the patient, left coronary injection significant narrowing of the trunk of the left coronal artery

tion in response to catecholamines and sympathetic $\alpha$-adrenergic receptor stimulation. The thyrotoxicosis is associated with increased sensitivity and numbers of adrenergic receptors. According to the ESC guidelines on vasospastic angina treatment, calcium channel blockers of the non-dihydropyridine class and long-acting nitroglycerin should be used. In the case of artery spasm in the progress of thyrotoxicosis, it is most important to achieve euthyroidism.

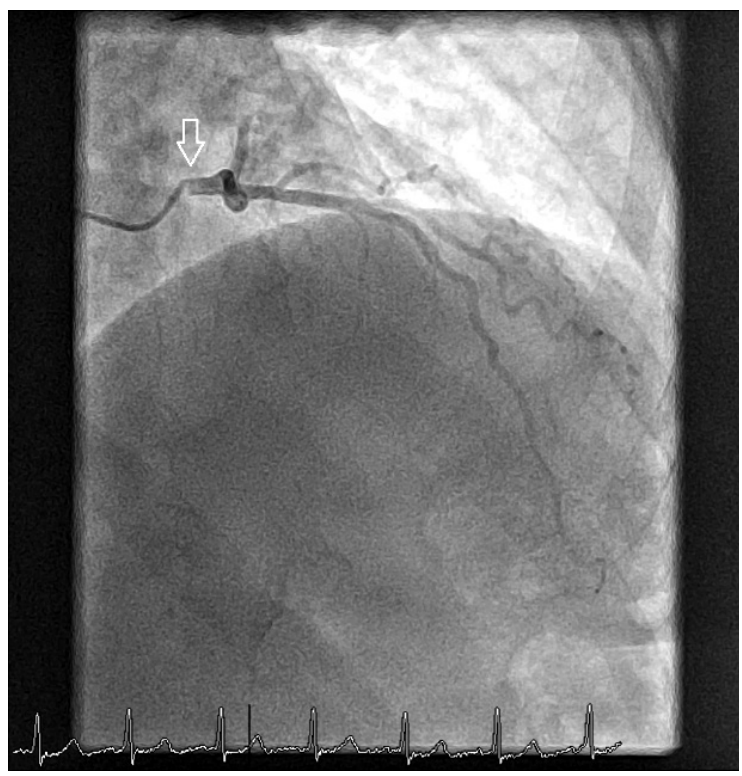

Figure 3. Coronary angiogram of the patient after administration of intra coronary nitroglycerin

\section{References}

1. Featherstone HJ, Stewart DK. Angina in ThyrotoxicosisThyroid-Related Coronary Artery Spasm. Arch Intern Med. 1983; 143(3): 554-555, doi: 10.1001/archinte.1983.00350030168028.

2. Chudleigh RA, Davies JS. Graves' thyrotoxicosis and coronary artery spasm. Postgrad Med J. 2007; 83(985): e5, doi: 10.1136/pgmj.2007.062299, indexed in Pubmed: 17989261.

3. European Society of Cardiology. 2020 Acute Coronary Syndromes (ACS) in Patients Presenting without Persistent ST-Segment Elevation (Management of) Guidelines. https://www.escardio.org/Guidelines/Clinical-Practice-Guidelines/Acute-Coronary-Syndromes-ACS-in-patients-presenting-without-persistent-ST-segm. 